\title{
Efficient Constant-Velocity Reconfiguration of Crystalline Robots*
}

\author{
Greg Aloupis ${ }^{\dagger} \quad$ Sébastien Collette ${ }^{\ddagger} \quad$ Mirela Damian ${ }^{\S}$ \\ Erik D. Demaine Dania El-Khechen $\|$ Robin Flatland ** \\ Stefan Langerman ${ }^{\dagger \dagger}$ Joseph O’Rourke ${ }^{\ddagger \ddagger}$ Val Pinciu ${ }_{\S} \S$ \\ Suneeta Ramaswami ศึ Vera Sacristán 1\|\| Stefanie Wuhrer ***
}

August 14, 2009

${ }^{*}$ A short version appeared at WAFR 2008 [Aloupis et al., 2008a], with title Realistic Reconfiguration of Crystalline (and Telecube) Robots

${ }^{\dagger}$ Université Libre de Bruxelles, Belgique, aloupis.greg@gmail.com. Supported by the Communauté française de Belgique - ARC.

${ }^{\ddagger}$ Chargé de Recherches du FNRS. Université Libre de Bruxelles, Belgique, sebastien.collette@ulb.ac.be.

$\S$ Villanova University, Villanova, USA, mirela.damian@villanova.edu.

"Massachusetts Institute of Technology, Cambridge, USA, edemaine@mit.edu. Partially supported by NSF CAREER award CCF-0347776, DOE grant DE-FG0204ER25647, and AFOSR grant FA9550-07-1-0538.

"Concordia University, Montreal, Canada, d_elkhec@cs.concordia.ca

** Siena College, Loudonville, N.Y., USA, flatland@siena.edu

${ }^{\dagger \dagger}$ Maître de Recherches du FRS-FNRS. Université Libre de Bruxelles, Belgique, stefan.langerman@ulb.ac.be.

${ }^{\ddagger \ddagger}$ Smith College, Northampton, USA, orourke@cs.smith.edu.

$\S \S$ Southern Connecticut State University, USA, pinciu@scsu.ctstateu.edu

ฯ Rutgers University, Camden, USA, rsuneeta@camden.rutgers.edu. Partially supported by NSF grant CCR-0204293.

"III Universitat Politècnica de Catalunya, Barcelona, Spain, vera.sacristan@upc.edu . Partially supported by projects MEC MTM2006-01267 and Gen. Cat. DGR 2009SGR1040.

***Carleton University, Ottawa, Canada, swuhrer@scs.carleton.ca 


\begin{abstract}
:
In this paper we propose novel algorithms for reconfiguring modular robots that are composed of $n$ atoms. Each atom has the shape of a unit cube and can expand/contract each face by half a unit, as well as attach to or detach from faces of neighboring atoms. For universal reconfiguration, atoms must be arranged in $2 \times 2 \times 2$ modules. We respect certain physical constraints: each atom reaches at most constant velocity and can displace at most a constant number of other atoms. We assume that one of the atoms has access to the coordinates of atoms in the target configuration.

Our algorithms involve a total of $O\left(n^{2}\right)$ atom operations, which are performed in $O(n)$ parallel steps. This improves on previous reconfiguration algorithms, which either use $O\left(n^{2}\right)$ parallel steps [Rus and Vona, 2001, Vassilvitskii et al., 2002, Butler and Rus, 2003] or do not respect the constraints mentioned above [Aloupis et al., 2009b]. In fact, in the setting considered, our algorithms are optimal. A further advantage of our algorithms is that reconfiguration can take place within the union of the source and target configuration space, and only requires local communication.
\end{abstract}

\title{
1 Introduction
}

Self-reconfiguring modular robots. Robots designed with inflexible structures tend to have a unique purpose. They can be very efficient but often lack versatility, in the sense that they might not perform unexpected tasks efficiently, or might have problems adapting to new environments.

For this reason, much research has been concentrated on the design of modular robots that can self-reconfigure. Modular robots are theoretically capable of reaching any shape that has the same mass/volume (restricted to the size of their finer components, or modules). Thus they not only become capable of seemingly limitless uses, but they also have the ability to self-repair (by replacing damaged modules), and navigate through new environments.

Several new prototypes of modular robots appear each year. Developers strive to solve hardware design challenges involving strength, precision, bonding, energy efficiency and flexibility of modules. An important goal is also to reduce module sizes. On the other hand, a significant problem in the field is to design efficient algorithms for self-reconfiguration. Various types of self-reconfiguring robots, as well as related algorithmic issues, are surveyed in [Murata and Kurokawa, 2007, Yim et al., 2007]. In this paper we focus on the (modular) Crystalline [Rus and Vona, 2001, Butler et al., 2002] and 
Telecube [Suh et al., 2002] robots, which are designed on a square lattice.

Crystalline and Telecube robots. The atoms of these robots are cubic in shape, and are arranged in a grid configuration. Each atom is equipped with mechanisms allowing it to extend each face out one unit and later retract it back. Furthermore, the faces can attach to or detach from faces of adjacent atoms; at all times, the atoms should form a connected unit. The default configuration for a Crystalline atom has expanded faces, while the default for a Telecube atom has contracted faces.

When groups of atoms perform the four basic atom operations (expand, contract, attach, detach) in a coordinated way, the atoms move relative to one another, resulting in a reconfiguration of the robot. Figure 1 shows an example of a reconfiguration.
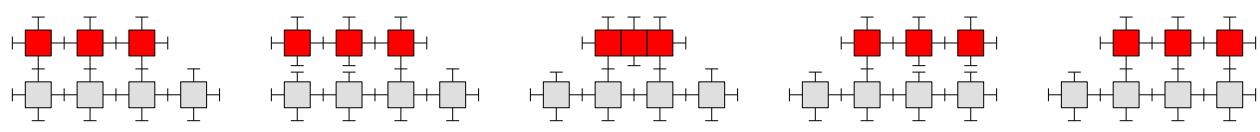

Figure 1: Example of reconfiguring Crystalline atoms.

To ensure that all reconfigurations are possible, atoms must be arranged in $k \times k \times k$ modules, where $k \geq 2$ [Aloupis et al., 2009b, Vassilvitskii et al., 2002]. In the $2 D$ setting that we focus on, we assume that modules consist of $2 \times 2$ atoms. Our algorithms can easily be extended to $3 D$.

We refer the reader to [Rus and Vona, 2001, Vassilvitskii et al., 2002, Aloupis et al., 2009b] for a more detailed introduction to these robots.

The model. The problem we solve is to reconfigure a given connected source configuration of $n$ modules to a specified, arbitrary, connected target configuration $T$ in $O(n)$ parallel steps. We allow modules to exert only a constant amount of force, independent of $n$. In particular, each module has the ability to push/pull one other module by a unit distance (the length of one module) within a unit of time. Simply bounding the force may still lead to arbitrarily high velocities and thus rather unrealistic motions. On the other hand, in some situations where maximal control is desired (e.g., treacherous conditions, dynamic obstacle environment, minimally stable static configuration of the robot itself) it may be desirable to strictly limit velocity. Thus we also bound maximum velocity (and so the momentum) by a constant (module length / unit time). Our algorithms are designed for Crystalline robots. In Section 5 we discuss how the algorithms can be 
adjusted to work for Telecube robots as well. In that section we also mention possible applications of our algorithms to robots made of other modules.

Related results. Algorithms for reconfiguring Crystalline and Telecube robots in $O\left(n^{2}\right)$ parallel steps have been given in [Rus and Vona, 2001, Vassilvitskii et al., 2002, Butler and Rus, 2003]. The same bound is implied in [Chirikjian et al., 1996], which deals with reconfigurations of a specific class of modular robots (more restrictive than Crystalline). An algorithm that uses $O(n)$ parallel steps for reconfiguring a robot within the bounding box of source and target configurations was given in [Aloupis et al., 2009b]. The total number of individual moves is also linear. However, no restrictions were made concerning physical properties of the robots. For example, $\Theta(n)$ strength is required, since modules can carry tall towers and push large masses during certain operations. An $O(\log n)$ parallel step algorithm for $2 \mathrm{D}$ robots that uses a total of $O(n \log n)$ atom moves and also stays within the bounding box is given in [Aloupis et al., 2008b], and this has recently been extended to 3D [Aloupis et al., 2009c]. However, in this algorithm, not only are modules assumed to have $\Theta(n)$ physical strength, but they can also reach $\Theta(n)$ velocity. An $O(\sqrt{n})$ time algorithm for $2 \mathrm{D}$ robots, using the third dimension as an intermediate, is given in [Reif and Slee, 2007]. This is optimal in the model considered, which permits linear velocities, but only constant acceleration. If applied within the model used in [Aloupis et al., 2008b], this algorithm would run in constant time. We remind the reader that, unlike [Reif and Slee, 2007, Aloupis et al., 2009b, Aloupis et al., 2008b], we limit force and velocity to a constant level.

Contributions of this paper. We present two algorithms to reconfigure Crystalline robots in $O(n)$ time steps, using $O(n)$ parallel moves per time step. Our first algorithm (Section 3 ) is slightly simpler to describe. It also forms the basis of our second algorithm (Section 4), which is exactly in-place, i.e., it uses only the cells of the union of the source and target configurations. This is particularly interesting if there are obstacles in the environment. Both algorithms consider the given robot as a spanning tree, and push leaves towards the root with "parallel tunneling". No global communication is required. This means that constant-size memory suffices for each non-root module, which can decide how to move at each step based solely on the states of its neighbors. In the realistic model considered in this paper, our algorithms are optimal, in the sense that certain reconfigurations require a linear number of parallel moves. Our first algorithm can be adapted for use 
with Telecube modules. However an adaptation of our in-place algorithm seems to require more memory for non-root modules. This is discussed in Section 5 .

\section{Primitive operations}

We restrict our descriptions to a $2 \mathrm{D}$ lattice whose cell size equals the size of one robot module (i.e., $2 \times 2$ connected atoms in their expanded state). None of our techniques depend on dimension, so it is straightforward to extend to 3 D robots. Given the $2 \times 2$ module size, a cell of the lattice can potentially contain two compressed modules (see Fig. 2b). Cells can be marked with an integer in $\{0,1,2\}$ : a 0 -cell corresponds to a node in $T$ that has no module yet, a 1-cell contains one module, and a 2-cell contains two (compressed) modules. In a 2-cell, we sometimes distinguish between the host module and the guest module: the host module is the one occupying the 1-cell prior to becoming a 2-cell; the guest module is the one compressing itself into the 1-cell occupied by the host module, thus turning the cell into a 2-cell (see Fig. 2a).

Let $r_{0}$ be a specialized module that has access to a map of the target configuration, $T$. We compute a spanning tree $S$ of the source configuration, rooted at $r_{0}$, and instruct modules to form attachments corresponding to tree edges in $S$. The spanning tree can be computed in linear time and constructed via local communication. The tree structure between cells is maintained throughout the algorithm by physical connections between host modules; note that guest modules are irrelevant in determining $S$. These modules are also responsible for the parent-child pointer structure of the tree. For each node $u \in S$, let $P(u)$ denote the parent of $u$ in $S$ (recall that both $u$ and $P(u)$ are host - not guest - modules). A child of a cell $u$ is adjacent either on the east, north, west, or south side of $u$. Let the highest priority child of $u$ be the first child in counterclockwise order starting with the east direction.

Let $m$ and $q$ be adjacent cells. We define the following primitive operations (illustrated in Fig. 2). A cell is meant to be engaged in at most one operation at any time.

1. $\operatorname{PushinLeaf}(m, q)$ - applies when $q=P(m), m$ is a leaf, and both are uncompressed. Here, $m$ becomes empty and $q$ becomes compressed (i.e., $q$ takes the module of $m$ as a guest). 
2. PopOutLeaf $(m, q)$ - applies when $q$ is compressed and $m$ is empty. This is the inverse of the PushInLeaf operation.

3. Transfer $(m, q)$ - applies when $m$ is compressed and $q$ is non-empty; if $q$ is compressed, the guests of both cells physically exchange positions. Otherwise, the guest of $m$ moves into (and becomes a guest of) $q$.

4. $\operatorname{Attach}(m, q)$ - applies when the host modules in $m$ and $q$ are unattached. The two modules make a physical connection.

5. $\operatorname{Detach}(m, q)$ - applies when the host modules in $m$ and $q$ are attached. The two modules break their physical connection.

6. $\operatorname{Switch}(m)-$ applies when $m$ is compressed. Its two modules physically switch positions (and roles of host and guest).

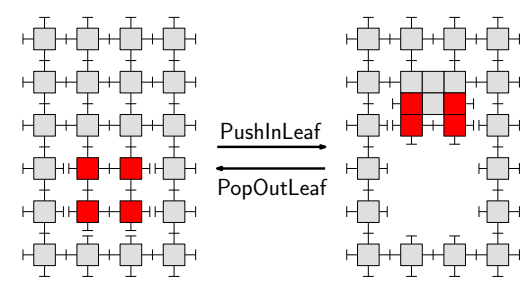

(a)

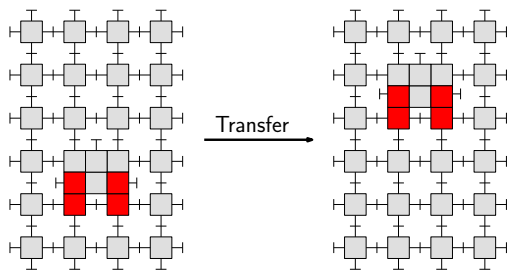

(c)

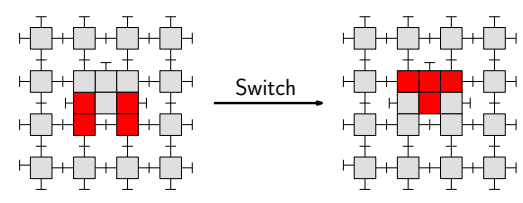

(b)

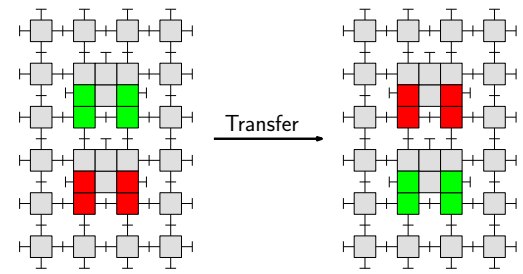

(d)

Figure 2: (a) PushInleaf and PopOutleaf. (b) Switch: guest and host exchange their roles and positions. (c) TRANSFER, when one module is compressed and the other is not. (d) TRAnsfer, when both modules are compressed. Only initial and final configurations are shown. Note that neighboring cells are not completely drawn.

In the remainder of this paper, we assume that all parallel motions are synchronized. However, due to the simple hierarchical tree structure of our robots, we find it plausible that our algorithms could be implemented so that modules may operate asynchronously. 
Lemma 1. Operations Pushinleaf, PopOutleaf, Switch and TransFER maintain the tree structure of a robot and can be executed in $O(1)$ time.

Proof. When each of the first three operations is performed individually, the robot remains connected. For example, in PushInLeaf $(m, q)$, the atoms of host module $q$ temporarily displace, in order to let those of $m$ occupy intermediate spaces. However, at all times one of the two atoms along every face of $q$ (other than the one adjacent to $m$ ) will remain in its original position so that connectivity is ensured; this property can be verified in Fig. 7 in the appendix. Note that the activity within guest module $m$ does not affect connectivity in the rest of the robot.

This temporary displacement can cause connectivity issues if modules neighboring the host module $q$ are also performing basic operations. To avoid any problems, we can subdivide one basic time unit into four subunits, so that each host module acts when it has the right parity of row and column. For example, in the first time subunit, modules located in (odd row, odd column) lattice cells are allowed to reconfigure; in the second time unit, (odd row, even column) modules reconfigure; and so on.

This way, when a leaf pushes into its parent, we can ensure that no other cells adjacent to the parent are active. This issue is even simpler to resolve in $3 \mathrm{D}$, where the actuation at the atomic level can be done by $2 \mathrm{D}$ layers.

Now consider the TRANSFER operation. In this operation, two adjacent cells interact and it is best not to let any of the six neighboring cells perform basic operations simultaneously. A similar lattice parity solution can be applied.

Regarding the tree structure, it is straightforward to see that SwITCH and Transfer only affect module positions, while PushInLeAf removes one leaf module from $S$ and PopOutLeAf adds one leaf module to $S$.

As for the complexity of the primitive operation, note that any permutation of atoms within a lattice cell containing two modules can be realized in linear time with respect to the size of the module (i.e., $O(1)$ time for our modules). Thus a compressed cell may transfer or push one module to any direction, and two modules within a cell can switch roles in $O(1)$ time.

In our basic motions, modules move by one unit length per time step. The two modules involved in a primitive operation do not carry other modules. Thus our reconfiguration algorithms place no additional force constraints beyond those required by any reconfiguration algorithm. 


\subsection{Details of the primitive operations}

Refer to Figures 7-11, located in the Appendix. Fig. 7 shows the finite sequence of basic atom operations (attach, detach, compress, expand) that implement PushInLeaf and PopOutLeaf, which are inverse operations.

Transfer $(m, q)$ can be described as a two-step operation. In the first step, module $m$ is rotated within its lattice cell, in order to face module $q$. We call this the positioning step. In the second step, the module $m$ is actually sent to the lattice cell of $q$. Depending on whether $q$ was compressed or uncompressed, we call this the send or the exchange step. Fig. 8 shows the finite sequence of basic atom operations (attach, detach, compress, expand) that produce the positioning step. Fig. 9 and Fig. 10 show the analogous sequence for the send and the exchange steps. TRANSFER is the result of appropriately concatenating these steps.

SWITCH is a particular permutation of atoms within a lattice cell containing two modules, which can be realized in linear time with respect to the size of the module. Fig. 11 shows the details of the SwITCH operation.

\section{Reconfiguration via canonical form}

This section describes an algorithm to reconfigure $S$ into $T$ via an intermediate canonical configuration. Modules follow a path directly to the root $r_{0}$, and into a canonical "storage configuration". We focus on the construction of one type of canonical form, a vertical line $V$. In fact $V$ could be any path that avoids the source configuration. Thus the entire reconfiguration can take place relatively close to the bounding box of $S$. Reconfiguring from $V$ to $T$ is nearly the inverse procedure and is relatively straightforward. Each module $m$ passes from the canonical form through $r_{0}$. It suffices for $r_{0}$ to provide $m$ with just a few bits of information, indicating where $m$ should have children. If we can afford to let $m$ store $O(\log n)$ bits, then we can even specify the size of the subtrees rooted at $m$ (this helps heuristically, and is described at the end of section 3). For this task, it is assumed that $r_{0}$ has access to a map of $T$ (perhaps stored in memory, or via direct communication with some external processor).

\subsection{Algorithmic details}

Our algorithm reconfigures $S$ into a vertical strip $V$ that begins at the maximum $y$-coordinate of $S$. We first move $r_{0}$ to a maximum possible $y$ coordinate: this involves pushing in a leaf and iteratively transferring it to 
$r_{0}$, so that $r_{0}$ becomes part of a 2-cell and is then able to iteratively transfer to a module of maximum $y$-coordinate. Note that this step might not be necessary in implementations in which all modules are capable of playing the role of $r_{0}$ (for example, if all modules have a map of $T$, or if all are capable of communicating to an external processor). This initial step is followed by two main phases, during which $r_{0}$ does not move.

In the first phase, we repeatedly apply procedure CLUSTERSTEP to move modules closer to $r_{0}$. This is done by compressing leaves into their parents, and moving up $S$ in parallel. The shape of $S$ shrinks during this procedure, as PushInLeaf operations in ClusterStep compress leaf modules into their parent cells. At the end of this phase, all non-leaf cells will become 2-cells. We refer to $S$ in this state as being fully compressed. It is not critical that all cells become compressed; in fact, we can proceed to the next phase as soon as the root becomes part of a 2-cell. The restriction for $S$ being fully compressed at the end of this phase will merely simplify our analysis of the total number of parallel steps in our algorithm.

\section{ClusterStep $(S)$}

For all cells $u$ in $S$ except for that containing $r_{0}$, execute the following in parallel: If $P(u)$ is a 1-cell

If $u$ is the highest priority child of $P(u)$ and all siblings of $u$ are leaves or 2-cells,

If $u$ is a 1-cell leaf then PushInLeaf $(u, P(u))$.

If $u$ is a 2 -cell, then Transfer $(u, P(u))$.

SourceCluster $(S)$
$\begin{gathered}\text { Repeat until } S \text { is fully compressed } \\ \text { ClusterStep }(S) .\end{gathered}$

The procedure SourceCluster is illustrated in Figure 3. The task of compressing a parent cell $P(u)$ falls onto its highest priority child, $u$. Note that $P(u)$ first becomes compressed only when all its subtrees are essentially compressed. That is, even if $u$ is ready to supply a module to $P(u)$, it waits until all other children are also ready. This rule could be altered, and in fact the whole process would then run slightly faster. Here, we ensure that once the root of a subtree becomes compressed, it will be able to supply a steady stream of guest modules to its ancestors. 

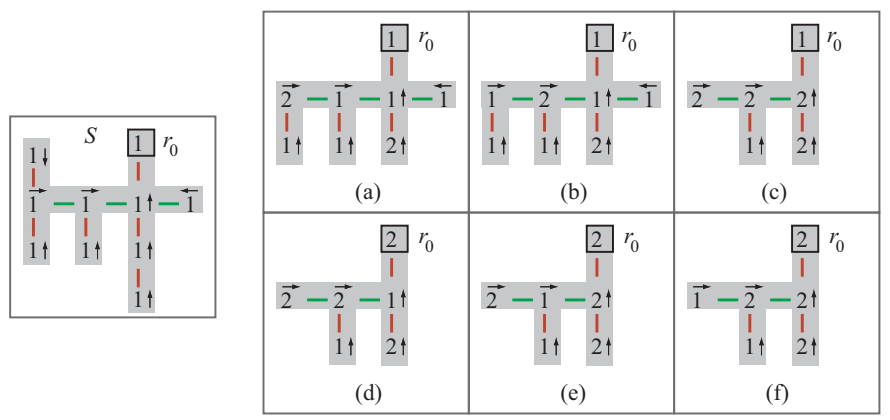

Figure 3: An example of SourceCluster. Arrows indicate the direction of the Transfer and PushInLeaf operations. After steps (a) through (e), the source configuration (left) becomes fully compressed (f).

In the second phase, we construct $V$ while emptying $S$, one module at a time. This is described in the second step of algorithm TreeToPATH, and is illustrated in Figure 4.

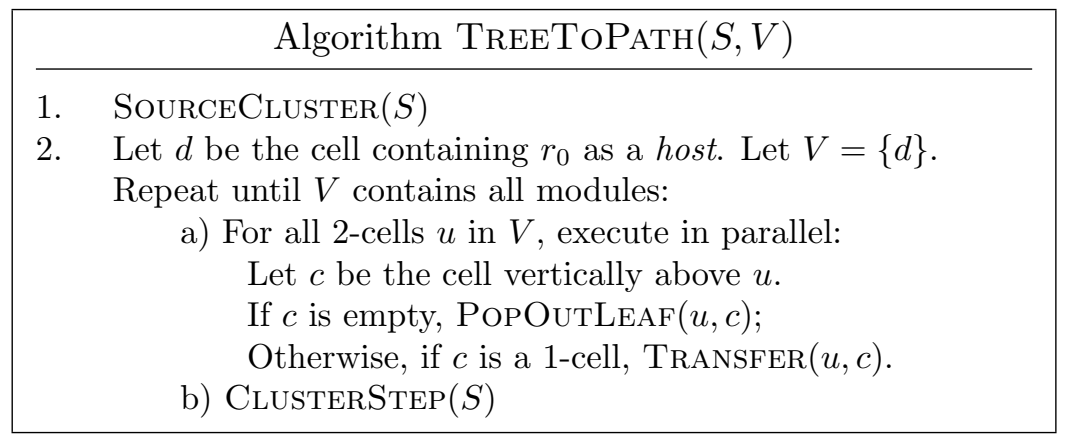

Lemma 2. If $S$ is a set of modules physically connected in a tree of cells, then ClusterSteP $(S)$ returns a tree containing the same set of modules, while maintaining connectivity. So does SourceCluster $(S)$.

Proof. Clusterstep invokes two basic operations, PushInLeaf and TrAnsfer. By Lemma 1, these operations independently maintain a tree. Since $u$ is involved in PushInLeaf or Transfer (with $P(u)$ ) only if it is a 1-cell leaf or a 2-cell respectively, but also only when $P(u)$ is a 1-cell, we know that $P(u)$ is not involved in any other such operation in parallel. Therefore every cell is involved in at most one basic operation at a time. The claim also follows immediately for SourceCluster. 

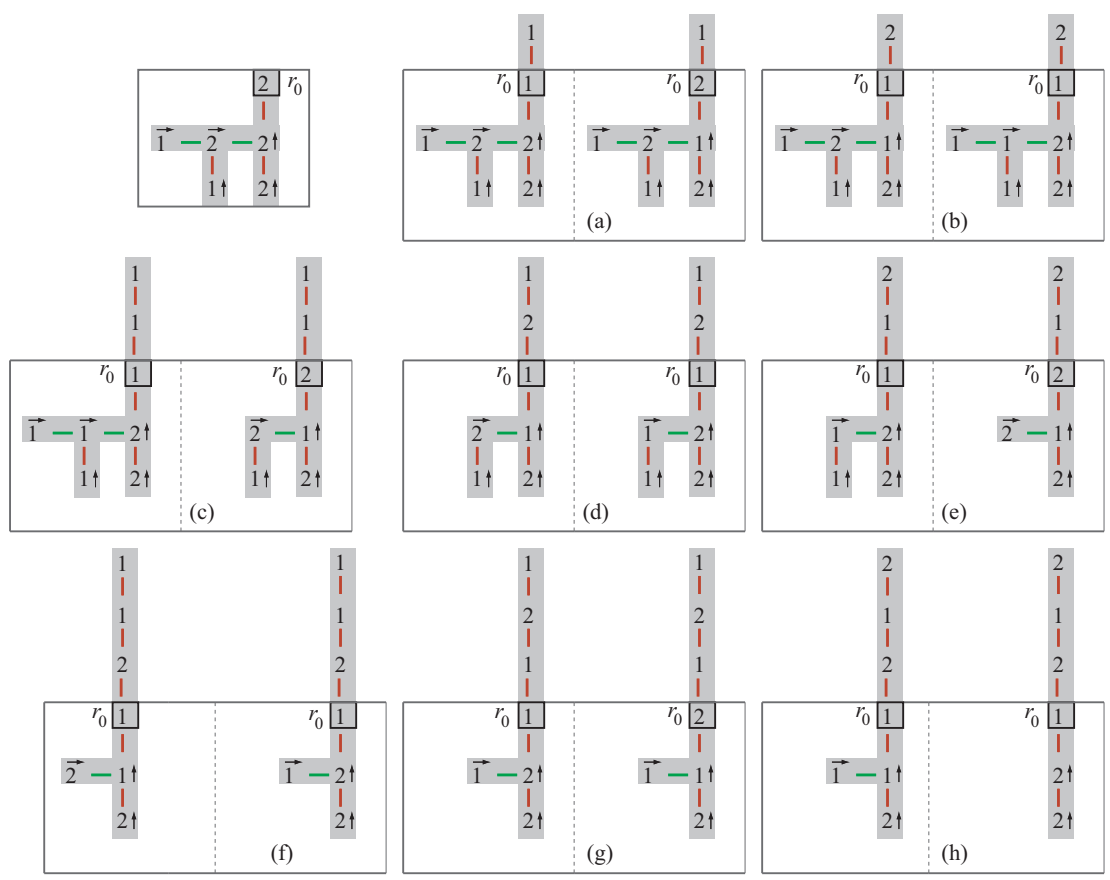

Figure 4: An example of TreeToPath. For each step, the two phases (PopOutleaf or Transfer, and ClusterStep) are shown. 
Define the height of a cell in $S$ to be the longest path to a leaf in its subtree, plus 1. By convention, leaves have height one.

Lemma 3. Let $r$ be a cell in $S$ with height $h \geq 2$. In iteration $h-1$ of SourceCluster $(S), r$ becomes a 2-cell for the first time.

Proof. Prior to the first iteration, $S$ contains only 1-cells. The proof is by induction on $h$. For the base case when $h=2$, the children of $r$ are leaves. Therefore, in the first iteration, during ClusterSTEP, the highest priority leaf compresses into $r$.

Now assume inductively that the lemma is true for all subtrees of height smaller than $h$. Cell $r$ must have at least one child $c$ with height $h-1$. By the inductive hypothesis, $c$ becomes a 2-cell in iteration $h-2$, and all of $r$ 's other non-leaf children are 2-cells by the end of iteration $h-2$. Therefore, at iteration $h-1$, for the first time the conditions are satisfied for $r$ to receive a module from its highest priority child during ClusterSteP.

Lemma 4. In iteration $i$ of SourceCluster, let $r$ be a 2-cell with height $h$ that transfers its guest module to $P(r)$. Then at the end of iteration $i+1$, $r$ is either a leaf or a 2-cell again.

Proof. First note that at the beginning of iteration $i+1, r$ is a 1-cell and $P(r)$ is a 2-cell. Thus if $r$ is a leaf after iteration $i$, it remains so. On the other hand if $r$ has children $(h \geq 2)$, it will become a 2-cell. We prove this by assuming inductively that our claim holds for all heights less than $h$. Consider the base case when $h=2$. At the end of iteration $i$, all children of $r$ are still leaves and thus one will compress into $r$ (note that $r$ might also become a leaf in this particular case).

For $h>2$, consider the iteration $j<i$ in which $r$ received the guest module that it later transfers to $P(r)$ in iteration $i$. At the beginning of iteration $j$, all of $r$ 's children were leaves or 2-cells, since that is a requirement for $r$ to receive a guest. Let $c$ be the child that passed the module to $r$. If $c$ used the PushInLeaf operation, then at the end of iteration $j, r$ has one fewer children (but at least one). The other children remain leaves or 2-cells until iteration $i+1$, when $r$ becomes a 1-cell again. Thus in iteration $i+1$, conditions are set for $r$ to receive a module.

On the other hand, if $c$ used the TRANSFER operation, we apply the inductive hypothesis: at the end of iteration $j+1 \leq i, c$ is either a leaf or a 2-cell. During iterations $j$ and $j+1$ in which $r$ is busy receiving or transferring a module, all other children of $r$ (if any) remain leaves or 2cells. Therefore in iteration $j+2 \leq i+1$, the conditions are set for $r$ to receive a module. 
Define the depth of a cell in a tree to be its distance from the root. Hence, the root has depth zero. Let the root of $S$ be at height $h_{0}$.

Lemma 5. SourceCluster terminates after at most $2 h_{0}-1$ iterations of ClusterStep.

Proof. We claim that at the completion of iteration $h_{0}-1+d$ of SourceCluster, all non-leaf modules at depth less than or equal to $d$ in $S$ are 2-cells (here we use $S$ to refer to the current instance of the dynamically changing tree, not the original $S$ ). The proof is by induction on $d$. The base case is the root of $S$ at depth $d=0$. By Lemma 3, the root becomes a 2-cell in iteration $h_{0}-1$. Assume inductively that our claim is true for all values $d^{\prime}$, where $0 \leq d^{\prime}<d$.

Now consider a cell $p$ at depth $d-1$ that has children. By the inductive hypothesis, $p$ and all its ancestors are 2-cells by the end of iteration $i=$ $h_{0}-1+(d-1)$, and $p$ is the last of this group to become a 2-cell. Thus at the beginning of iteration $i$, all children of $p$ are either leaves or 2-cells. During iteration $i$, only $p$ 's highest priority child $c$ changes, either by transferring a guest module into $p$ (if $c$ is a 2-cell), or by pushing into $p$ (if $c$ is a 1-cell leaf). In the first case, by Lemma $4, c$ will be a 2 -cell or a leaf by the end of iteration $i+1$. In the second case, $c$ is not part of $S$ anymore.

Since $p$ will not accept new guest modules after iteration $i$ (because all its ancestors are 2-cells), all siblings of $c$ remain leaves or 2-cells during iteration $i+1$. Thus at the end of this iteration, our claim holds for depth $d$. By setting $d=h_{0}$, our result follows.

Let a long gap consist of two adjacent 1-cells that are not leaves. A tree is root-clustered if it has no long gaps. Observe that a fully compressed tree is a special case of a root-clustered tree.

Lemma 6. Let $S$ be a root-clustered tree. Then after one application of ClusterStep $(S), S$ remains root-clustered.

Proof. This follows from claims in the proof of Lemma 4. Specifically, consider any 2-cell $u$. If CLUSTERSTEP keeps $u$ as a 2-cell, then $u$ is not part of a long gap. Otherwise, if $u$ sends a module to $P(u)$, none of the children of $u$ attempt to transfer a module to $u$. Now consider any 1-cell non-leaf child $y$ of $u$. Since there was no long gap in $S$, all children of $y$ were either 2-cells or leaves. Thus $y$ will become a 2-cell during this iteration of CLUSTERSTEP. Again we conclude that $u$ cannot be part of a long gap.

Theorem 1. Algorithm TreeToPATH terminates in linear time. 
Proof. By Lemma 5, SourceCluster terminates in linear time. In fact by treating the final top position of $V$ as an implicit root, our claim follows.

More specifically, however, we analyze the transition from $S$ into $V$. When SourceCluster terminates, $S$ is fully compressed (i.e., rootclustered), and we set $r_{0}$ to be the host in cell $d$.

First we mention that all basic operations are performed legally, that is, every cell is involved in at most one operation. In step 2a, every 2-cell is involved in an operation, only if the cell above it is not a 2-cell. Therefore both cells are only involved in this operation. Step $2 b$ is safe, by Lemma 2 .

In step $2 \mathrm{a}, d$ sends a module to the empty position $c$ vertically above, if $c$ is not a 2-cell. We may treat the position $c$ as $P(d)$, and consider step 2a to be synchronous to step $2 \mathrm{~b}$. In other words, $d$ is the only child of $c$, and thus $d$ follows the same rules as CLusterSTEP. In fact, since $S$ is fully compressed, after the first iteration of phase 2 , the tree rooted at $c$ will be root-clustered (only $c$ and the highest-priority child of $d$ will not be 2-cells). Therefore, by Lemma 6 , in every iteration of phase $2, S$ remains root-clustered. Thus in every even iteration, $d$ supplies a module to $c$, and in every odd iteration $d$ is given a module from one of its children. Informally, when $d$ sends a module up into $V$, the gap (in the sense of lack of guest module) that is created in $S$ travels down the highest priority path of $S$ until it disappears at a leaf. In general, a guest module on the priority path will never be more than two steps away from $d$, following the analysis of Lemma 4 . Within $V$, a stream of guest modules, two units apart, will move upward. One module will pop up into an empty cell, every three iterations. Thus compressed modules in $V$ are always able to progress.

We now briefly discuss the reconfiguration from $V$ to $T$. If we merely wish to construct the shape of $T$, then we can assume that $V$ does not intersect the cells that will be occupied by $T$. Otherwise, if $T$ must occupy specific cell coordinates, it is trivial to move $V$ to a position where our assumption will hold.

For the construction of $T$, let us first assume that the memory of each module suffices to count to $n$. All modules from $V$ pass through the root $r_{0}$ on their way to $T$. Once a module $m$ reaches $r_{0}$, the root determines the position of $m$ in $T$ and supplies $m$ with three values corresponding to the sizes of the three subtrees of $m$ in $T$. Then $r_{0}$ transfers $m$ to the highestpriority child $c$ of $r_{0}$ whose subtree is not full yet. The child $c$ in turn transfers $m$ to its own highest-priority child not yet full and so on, until $m$ encounters the conditions to pop into an empty cell in $T$, as directed by its host module. From that point on, $m$ simply awaits modules transferred by 
its parent and directs them to its children according to our priority rules (counterclockwise starting with the east child). In order to decide where to send an incoming module, $m$ keeps count of all modules passed through; this information, along with the information collected from $r_{0}$ (the sizes of its three subtrees), suffices for $m$ to avoid sending an incoming module into a completed subtree.

If we do not have the luxury of allowing modules to count, we do the following. The root does not tell $m$ the size of its subtrees, but instead it just tells $m$ if it will have a subtree, in each direction. Priority rules are followed, as before. The only difference is that when $m$ reaches its final position, it will not be able to determine when its subtrees are full. Thus each module performs an entire Depth-First Search of the partial structure of $T$. This involves backtracking, which can be dealt with via TrAnsfer. We omit details here, since this backtracking issue appears again and is clarified in section 4 .

We remind the reader that our first phase need not terminate before the second commences. By compressing leaves and sending them towards the root, while simultaneously constructing $V$ from the root whenever it becomes compressed, the target configuration will be constructed even faster. Splitting into two distinct phases simply helps with the analysis.

\section{In-place reconfiguration}

This section describes an in-place algorithm that reconfigures $S$ into $T$ by restricting the movement of all modules to the space occupied by $S \cup T$, as long as they intersect. If $S$ and $T$ do not intersect, then we also use the cells on the shortest path between them. Our description assumes intersection. If all modules were to know which direction to take in each time unit (for example, by having an external source synchronously transmit instructions to each module individually), then it would not be difficult to design an in-place algorithm similar to the one in Section 3. However, since we impose the restriction that all modules are only capable of communicating locally, it is up to $r_{0}$ to direct all action.

\subsection{Overview}

Our algorithm consists of two phases. The first phase is identical to phase 1 of the TreeToPATH algorithm from Section 3 (i.e., clustering around the root). 
In the second phase, $r_{0}$ carries out a DFS (depth-first search) walk on $T$, dynamically constructing portions that are not already in place. Apart from modules in cells adjacent to $r_{0}$ that receive its instructions, all other modules simply try to keep up with $r_{0}$ (i.e., they follow ClusterStep). Note that if $r_{0}$ is not initially inside $T$, it first must travel to such a position. At any time, this "moving root" will either be traveling through modules that belong to the partially constructed tree $T$, or will be expanding $T$

beyond the current tree structure, using compressed modules that are tagging along close to $r_{0}$.

\subsection{Algorithmic details}

The InPlaceRECONFIGURATION algorithm maintains a dynamically changing tree $S$, and a connected subset $S_{\ell}$ of that tree. Each cell $u$ maintains two links: a physical link corresponding to the physical connection between $u$ and $P(u)$ in $S$, and a logical link that is only present between adjacent nodes in $S_{\ell}$. We call the tree $S_{\ell}$ induced by the logical links the logical tree.

Tree $S$ always contains all occupied cells. Tree $S_{\ell}$ is the smallest subtree of $S$ that contains modules not in their final position in $T$. Thus at the end of the algorithm, $S=T$ and $S_{\ell}=\emptyset$.

The full algorithm is summarized in the following:

\begin{tabular}{|c|c|}
\hline \multicolumn{2}{|r|}{ Algorithm InPlaceReCONFIguRation $(S, T)$} \\
\hline Phase 1. & $S \leftarrow \operatorname{SourceCluster}(S)$ \\
\hline Phase 2. & $\begin{array}{l}S_{\ell} \leftarrow S \text {. } \\
\text { Repeat until } r_{0} \text { reaches the final position in its DFS traversal: } \\
\qquad S \leftarrow \text { TARGETGROW }(S, T) \text {. }\end{array}$ \\
\hline
\end{tabular}

We continue with a description of the operation of TARGETGROW. 


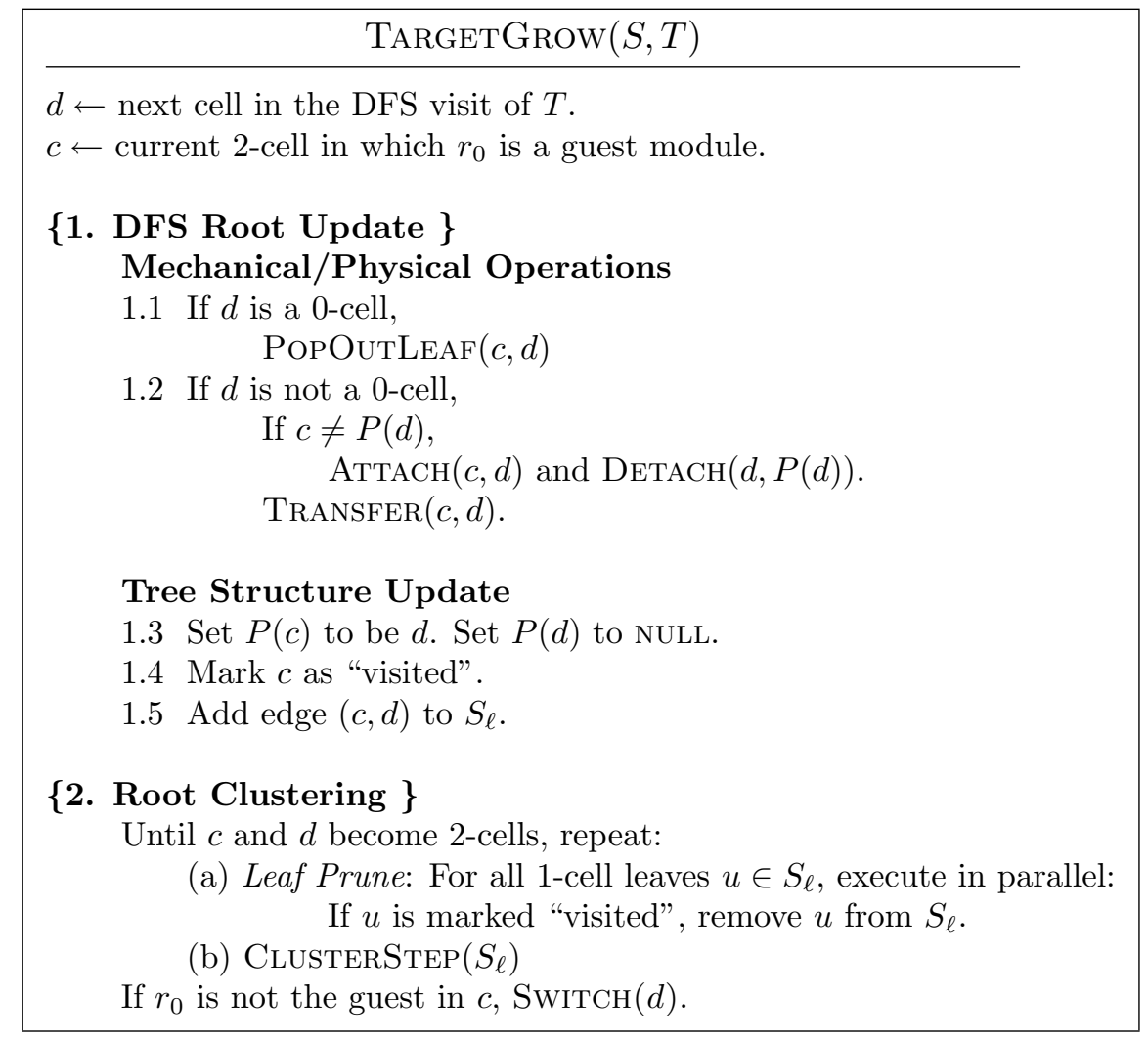

Note that in Phase 2, we start with $S_{\ell}=S$. Throughout this phase, the structure of $S_{\ell}$ is identical to the structure of $S$, with the exception that some branches of the tree $S_{\ell}$ are logically trimmed off through iterative leaf prune operations. The host module in each cell uses a bit to determine if the cell is also part of $S_{\ell}$. Pointers between cells and their parents apply for both trees.

The main idea of the target growing phase is to move $r_{0}$ through the cells of $T$ in a DFS order. A caravan of modules will follow $r_{0}$, providing a steady stream of modules to fill in empty target cells that $r_{0}$ encounters. The algorithm repeats the following main steps:

1. DFS Root Update: $r_{0}$ is the guest of 2-cell $c$ and is ready to depart. It marks $c$ as "visited" (i.e., $c$ now belongs to $T$ ). Then $r_{0}$ moves to the next cell $d$ encountered in a DFS walk of $T$. This is accomplished either by uncompressing (popping) $r_{0}$ into $d$ (see Fig. $5(\mathrm{a} \rightarrow \mathrm{b})$ ), or by transferring $r_{0}$ to $d$ (see Fig. $5(\mathrm{e} \rightarrow \mathrm{f})$ ). Cell $d$ is added to $S_{\ell}$, if not already included. 
2. Root Clustering: Modules in $S_{\ell}$ attempt to move closer to $r_{0}$, to ensure that they are readily available when $r_{0}$ needs them. However, host modules in their final target position should never be displaced from that position, so we must carefully prevent such modules from compressing towards $r_{0}$. To achieve this, we alternate between the following two steps, until $c$ and $d$ both become 2-cells:

(a) Logical Leaf Pruning: remove any 1-cell leaf of $S_{\ell}$ that has been visited (i.e., is in $T$ ). Note that a pruned 1-cell may end up back in $S_{\ell}$ one more time, during Root Update.

(b) Cluster Step: this step is applied to $S_{\ell}$. Thus, only modules that are guests or unvisited leaves will try to move towards $r_{0}$.

Fig. 5 illustrates the InPlaceRECONFIGURATION algorithm with the help of a simple example.

\subsection{Algorithm correctness and complexity}

Lemma 7. Algorithm InPlaceReCONFIGURATIOn maintains a physically connected tree that contains all modules.

Proof. By Lemma 2, SourceCluster produces a tree $S$ containing all robot modules. Thus we must show that TARGETGROw maintains such a tree when it receives one as input. We first analyze step 1 (DFS root update). In step 1.1, PopOutLeaf maintains a tree, by Lemma 1. In step $1.2, d$ is already part of $S$. Now if $c \neq P(d)$, we attach $c$ to $d$, which creates a cycle. However, we immediately break this cycle by detaching $d$ from $P(d)$, and thus $S$ is restored to a tree. See Figs. $5(c \rightarrow d \rightarrow e)$ for an example. Regardless of the initial relationship between $c$ and $P(d)$, and the possible re-structuring of $S$, we proceed with $\operatorname{TRANSFER}(c, d)$, which maintains tree connectivity, by Lemma 1 .

After steps 1.1 and 1.2 or the DFS update, no other physical connections are altered. Pointers are modified to reflect the physical changes made. Since the root acts alone, our assumption that every cell is involved in one operation at a time holds.

Finally, $S$ remains a physical tree during step 2 of TARGETGrow. This basically follows from two observations: (a) step $2 a$ changes only the logical tree $S_{\ell}$, and (b) ClusterStep maintains a tree, by Lemma 2. The only issue remaining is to prove that whenever a 1-cell leaf becomes involved in Pushinleaf in Clusterstep, it is not visited. Not only do we wish to avoid moving visited 1-cells, but this operation could cause a physical 


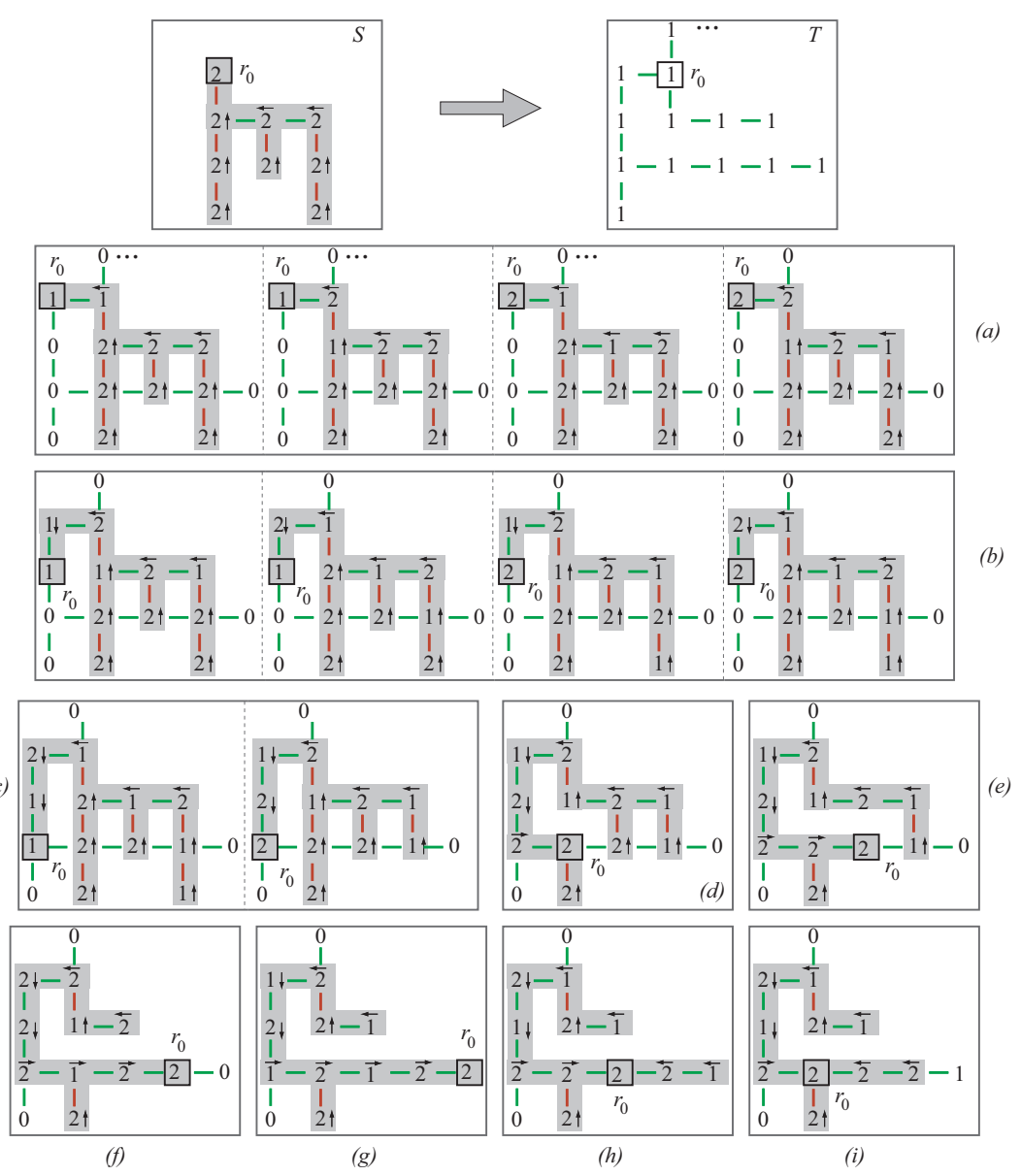

Figure 5: Reconfiguring $S$ into $T$ : the top row shows $S$ (after SourceClusTER) and $T$. Links in $S_{\ell}$, which is shaded, are depicted as arrows. Subsequent figures show $S$ (with its logical subtree $S_{\ell}$ shaded) (a) after TARGETGrow, with each intermediate step illustrated (DFS root update on the left and the subsequent 3 clustering steps on the right); (b) after TARGETGROW, with each intermediate step illustrated; (c) after TARGETGRow, with its two main steps (root update and root clustering) illustrated; $(d, e, f, g)$ show the next 4 TARGETGROW steps; ( $h$ ) after the next 2 TARGETGROW steps; (i) after the next TARGETGROW (note the rightmost 1-cell leaf getting disconnected from $S_{\ell}$ ); the process continues. 
disconnection if $S \neq S_{\ell}$. It suffices to claim that $S_{\ell}$ never contains a visited 1-cell leaf. This is in accordance to our definition of $S_{\ell}$ being the smallest tree containing unvisited modules. Given such a structure, the following situations can arise for a leaf $u$ in step 2. The leaf $u$ of $S_{\ell}$ could remain unaltered, or if it is a 1-cell (meaning, not visited) it could use PUSHINLEAF to become the guest in a new 2-cell leaf. Finally if $u$ is a 2-cell and becomes a 1-cell, we prune the 1-cell from $S_{\ell}$ if it is visited.

In phase 1 of InPlaceReconfiguration, the SourceCluster call produces a root-clustered tree containing $r_{0}$ in a 2-cell. We now show that phase 2 maintains this property in constant time, regardless of how $r_{0}$ moves.

Lemma 8. TARGETGROW maintains $S_{\ell}$ as a root-clustered tree containing $r_{0}$ in a 2-cell. Furthermore, the procedure uses $O(1)$ parallel steps.

Proof. The proof is rather similar to that of Lemma 6. Since the structure of $S_{\ell}$ is identical to the structure of $S$, with the exception of some branches being trimmed off, it follows from Lemma 7 that $S_{\ell}$ is physically connected. This property can also be derived from the fact that a cell $d$ is attached to one node only in $S_{\ell}$, thus never creating a cycle.

Let $S_{\ell}^{i}$ denote the root-clustered tree that is input for TARGETGRow at iteration $i$. In step 1 (DFS root update), $S_{\ell}^{i}$ will be modified according to any physical operations carried out (PopOutLeAf and Transfer ). By Lemma 7 , these changes result in a tree, which we call $S_{\ell}^{i+1}$.

Since step 1 only affects $c$ and $d$, it follows that at the beginning of step 2, a long gap in $S_{\ell}^{i+1}$ must contain $c$, which becomes a 1-cell via POPOUTLEAF (see Fig. 6a), or via Transfer (see Fig. 6b).

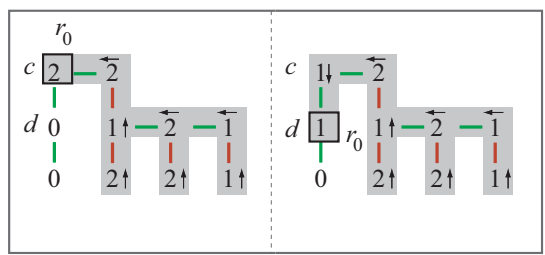

(a)

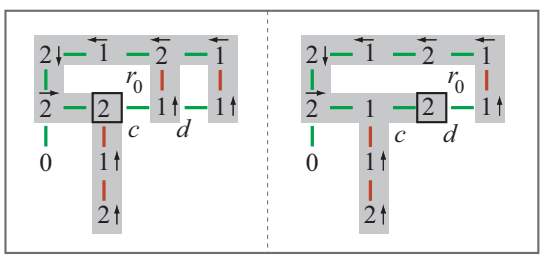

(b)

Figure 6: DFS Root update (a) $\operatorname{PopOutLeaf}(c, d)(\mathrm{b}) \operatorname{Transfer}(c, d)$.

We now show that the loop in step 2 of TARGETGRow iterates at most four times before our claim holds. Recall that, since $S_{\ell}^{i}$ was root-clustered, children of $c$ are either leaves, 2-cells, or their children have that property.

Any Leaf Prune operation only trims visited 1-cell leaves from the tree and thus does not affect the root-clustered property of the tree. There 
are two cases for the number of CLUSTERSTEP applications required to terminate the loop:

1. $S_{\ell}^{i+1}$ was obtained via PopOutLeaf (step 1.1): In this case $c$ and $d$ are 1-cells at the beginning of step 2. If all children of $c$ are leaves or 2-cells, then in the first iteration of CLusterSteP, $c$ will become a 2-cell again. Otherwise, since $S_{\ell}^{i}$ was root-clustered, any non-leaf 1-cell child will become a 2-cell in the first iteration. Thus in the second iteration at the latest, $c$ will become a 2-cell. Furthermore, just as described in Lemma 6, the subtree rooted at any child of $c$ remains root-clustered after the first application of CLUSTERSTEP (in particular, for the highest-priority child which is the only one that changes). Similarly, by the time $c$ becomes a 2-cell, the subtree rooted at $c$ also becomes root-clustered. The third ClusterSteP makes $d$ a 2-cell root of a root-clustered tree, since all children of $c$ must have been leaves or 2-cells to supply a module to $c$. The fourth ClusterSteP makes $c$ a 2-cell, which terminates the loop.

2. $S_{\ell}^{i+1}$ was obtained via TrAnsfer (step 1.2): In this case $d$ is already a 2-cell at the beginning of step 2 because of the TRANSFER operation in step 1.2. If $c$ remains a 2 -cell during the transfer, then $S_{\ell}^{i+1}$ is already root-clustered and the loop condition is satisfied. If $c$ is a 1-cell, arguments similar to case 1 imply that after one application of ClusterStep, $S_{\ell}^{i+1}$ is root-clustered. A second application of ClusTERSTEP makes $c$ a 2-cell, which terminates the loop.

This concludes the proof.

Theorem 2. The InPlaceReCONFIGURATION algorithm can be implemented in $O(n)$ parallel steps.

Proof. By Lemma 5, phase 1 uses $O(n)$ steps. Step 2 of InPlaceReconfigURATION has $O(n)$ iterations, since DFS has $O(n)$ complexity. By Lemma 8, each iteration takes constant time.

\section{Observations}

Matching lower bound: Transforming a horizontal line of modules to a vertical line requires a linear number of parallel steps, if each module can 
only displace one other module and maximum velocity is constant.

3D: All of our techniques apply directly to $3 D$ robots, once the top and bottom sides of cells are incorporated into our highest priority rule.

Reconfiguration of labeled robots: Our algorithms are essentially unaffected if labels are assigned to modules. This is of interest if a robot is to have specialized modules, equipped with cameras, drills, etc. In TREEToPATH, assume that the partially constructed canonical path is sorted. Then a new module $m$ can bubble/tunnel to its position by successive applications of the TRANSFER primitive operation. When it gets there, the tail of the path (from $m$ to leaf) must shift over. This is straightforward, involving propagation of one compressed unit, and does not interfere with other modules following $m$. At all times, $m$ or its replacement makes steady progress towards the leaf.

For the in-place algorithm, $T$ can first be constructed disregarding labels. A similar type of bubble-sort can then be applied, taking place within $T$.

Telecube robots: The natural state of a telecube robot has atom arms contracted. There is no room to compress two modules into one cell. Thus an algorithm cannot commence with PUSHINLEAF operations, and it is not possible to physically exchange modules in adjacent cells while remaining in place. However, consider our first algorithm. We do not even need a SourceCluster phase, since all atoms are packed together. The root can transmit an instruction to a cell at maximum $y$-coordinate to act as root and immediately push out two of its atoms. For the construction of $V$, all analysis follows. It seems that labeled atoms within a module might become separated (for example, if the module is at a junction in a tree). Thus an extra step is used, to collect the root atoms at the bottom of $V$.

Exact in-place reconfiguration is impossible for telecube robots if the modules are labeled. Thus the root cannot travel to any position within $S$. It might be possible to deal with this issue by requiring larger modules and designing a "reduced module shape" for the root (e.g., fewer atoms, using naturally expanded links). Instead, we could require that all modules have access to the map of $T$, which means any module can begin to expand $T$ by filling adjacent 0 -cells. Instead of backtracking or advancing through non-empty cells of $T$ physically, the root can just tell its neighbors to take over. Eventually a new root module would expand $T$ at a different connected component of 0-cells.

Other modular robots: It has been shown [Aloupis et al., 2009a] that suitably constructed modules of other prototypes (e.g., M- 
TRAN [Kurokawa et al., 2007], ATRON [Jørgensen et al., 2004]) are capable of simulating Crystalline atoms. Such modules may require 50-100 atoms to simulate one Crystalline atom, and the resulting shape is not compact. Nevertheless, the result in [Aloupis et al., 2009a] implies that our results here apply to large systems of other prototypes.

Acknowledgments. We thank the other participants of the 2008 Workshop on Reconfiguration at the Bellairs Research Institute of McGill University for providing a stimulating research environment. We also thank the reviewers of [Aloupis et al., 2008a] for their constructive comments.

\section{References}

[Aloupis et al., 2009a] Aloupis, G., Benbernou, N., Damian, M., Demaine, E., Flatland, R., Iacono, J., and Wuhrer, S. (2009a). Efficient reconfiguration of lattice-based modular robots. In European Conference on Mobile Robots (accepted).

[Aloupis et al., 2008a] Aloupis, G., Collette, S., Damian, M., Demaine, E. D., El-Khechen, D., Flatland, R., Langerman, S., O'Rourke, J., Pinciu, V., Ramaswami, S., Sacristán, V., and Wuhrer, S. (2008a). Realistic reconfiguration of Crystalline and Telecube robots. In 8th International Workshop on the Algorithmic Foundations of Robotics (WAFR).

[Aloupis et al., 2009b] Aloupis, G., Collette, S., Damian, M., Demaine, E. D., Flatland, R., Langerman, S., O'Rourke, J., Ramaswami, S., Sacristán, V., and Wuhrer, S. (2009b). Linear reconfiguration of cube-style modular robots. Computational Geometry: Theory and Applications, 42(6-7):652-663.

[Aloupis et al., 2008b] Aloupis, G., Collette, S., Demaine, E. D., Langerman, S., Sacristán, V., and Wuhrer, S. (2008b). Reconfiguration of cubestyle modular robots using $O(\log n)$ parallel moves. In Proc. Intl. Symp. on Algorithms and Computation (ISAAC 2008), volume 5369 of LNCS.

[Aloupis et al., 2009c] Aloupis, G., Collette, S., Demaine, E. D., Langerman, S., Sacristán, V., and Wuhrer, S. (2009c). Reconfiguration of 3D Crystalline robots using $O(\log n)$ parallel moves. Computational Geometry: Theory and Applications (submitted). 
[Butler et al., 2002] Butler, Z., Fitch, R., and Rus, D. (2002). Distributed control for unit-compressible robots: Goal-recognition, locomotion and splitting. IEEE/ASME Trans. on Mechatronics, 7(4):418-430.

[Butler and Rus, 2003] Butler, Z. and Rus, D. (2003). Distributed planning and control for modular robots with unit-compressible modules. Intl. Journal of Robotics Research, 22(9):699-715.

[Chirikjian et al., 1996] Chirikjian, G., Pamecha, A., and Ebert-Uphoff, I. (1996). Evaluating efficiency of self-reconfiguration in a class of modular robots. Journal of Robotic Systems, 13(5):317-338.

[Jørgensen et al., 2004] Jørgensen, M. W., Østergaard, E. H., and Lund, H. H. (2004). Modular ATRON: Modules for a self-reconfigurable robot. In Proc. of the International Conference on Intelligient Robots and Systems, pages 2068-2073.

[Kurokawa et al., 2007] Kurokawa, H., Tomita, K., Kamimura, A., Kokaji, S., Hasuo, T., and Murata, S. (2007). Self-reconfigurable modular robot m-tran: distributed control and communication. In RoboComm '07: Proceedings of the 1st international conference on Robot communication and coordination, pages 1-7, Piscataway, NJ, USA. IEEE Press.

[Murata and Kurokawa, 2007] Murata, S. and Kurokawa, H. (2007). Selfreconfigurable robots: Shape-changing cellular robots can exceed conventional robot flexibility. IEEE Robotics \& Automation Magazine, 14(1):4352 .

[Reif and Slee, 2007] Reif, J. H. and Slee, S. (2007). Optimal kinodynamic motion planning for self-reconfigurable robots between arbitrary 2D configurations. In Robotics: Science and Systems Conference, Georgia Institute of Technology.

[Rus and Vona, 2001] Rus, D. and Vona, M. (2001). Crystalline robots: Self-reconfiguration with compressible unit modules. Autonomous Robots, 10(1):107-124.

[Suh et al., 2002] Suh, J. W., Homans, S. B., and Yim, M. (2002). Telecubes: Mechanical design of a module for self-reconfigurable robotics. In Proc. of the IEEE Intl. Conf. on Robotics and Automation, pages 40954101. 
[Vassilvitskii et al., 2002] Vassilvitskii, S., Yim, M., and Suh, J. (2002). A complete, local and parallel reconfiguration algorithm for cube style modular robots. In Proc. of the IEEE Intl. Conf. on Robotics and Automation, pages $117-122$.

[Yim et al., 2007] Yim, M., Shen, W.-M., Salemi, B., Rus, D., Moll, M., Lipson, H., Klavins, E., and Chirikjian, G. S. (2007). Modular selfreconfigurable robots systems: Challenges and opportunities for the future. IEEE Robotics \& Automation Magazine, 14(1):43-52. 


\section{Appendix}

Pushinleaf and PopOutleaf

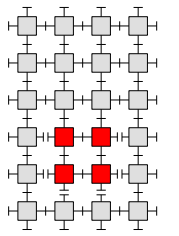

(a)

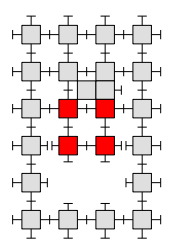

(f)

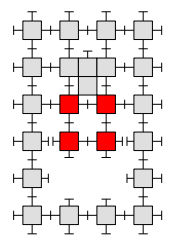

(k)

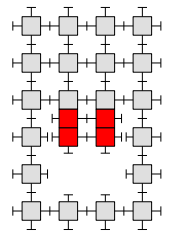

(b)

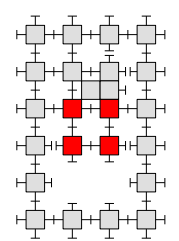

(g)

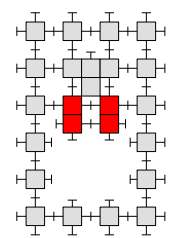

(l)

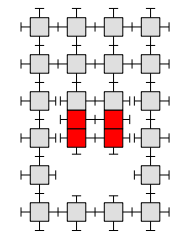

(c)

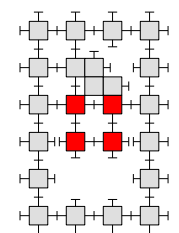

(h)

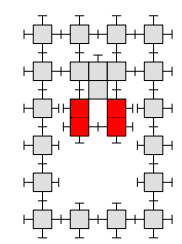

(m)

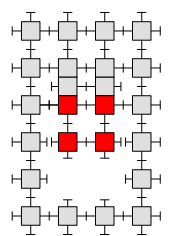

(d)

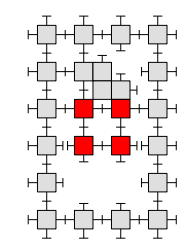

(i)

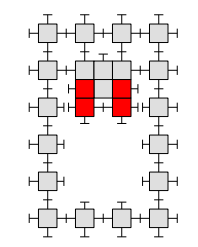

(n)

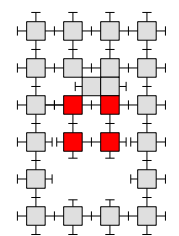

(e)

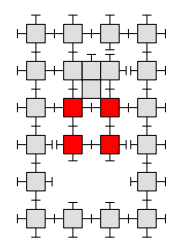

(j)

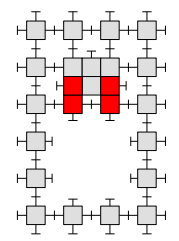

(o)

Figure 7: Details of PushInLeaf and PopOutLeaf. 
TRAnsfer

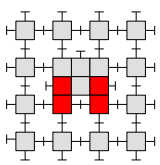

(a)

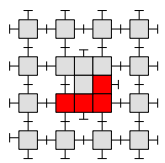

(f)

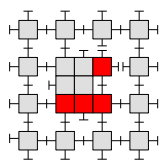

$(\mathrm{k})$

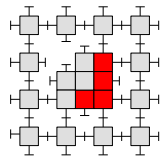

(p)

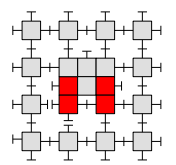

(b)

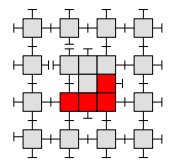

(g)

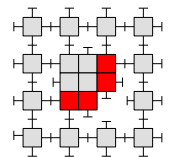

(l)

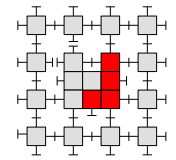

(q)

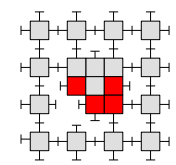

(c)

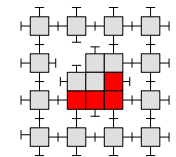

(h)

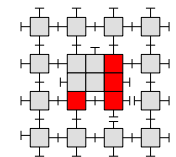

(m)

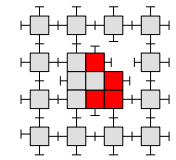

(r)

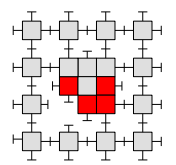

(d)

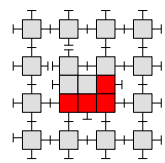

(i)

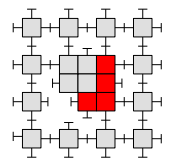

(n)

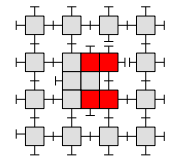

(s)

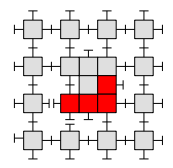

(e)

Figure 8: Details of the positioning step of the primitive operation $\operatorname{Transfer}(m, q)$. Before being sent to the lattice position of $q$, the module $m$ is rotated within its lattice cell, in order to face $q$. 


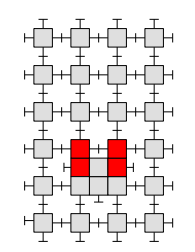

(a)

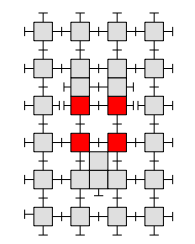

(f)

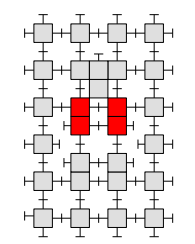

(k)

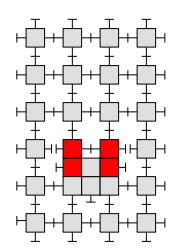

(b)

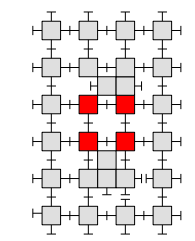

(g)

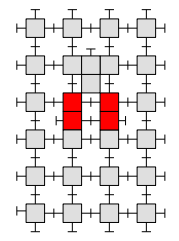

(1)

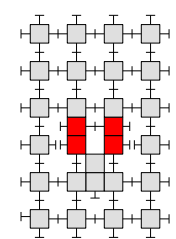

(c)

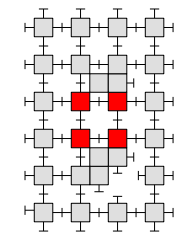

(h)

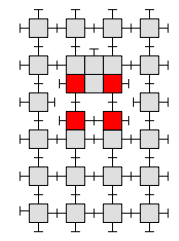

(m)

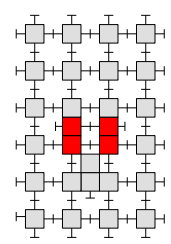

(d)

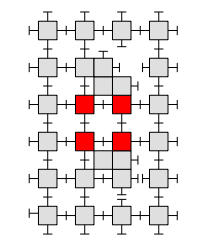

(i)

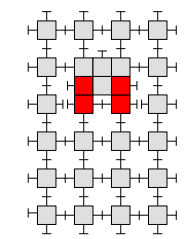

(n)

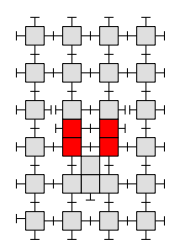

(e)

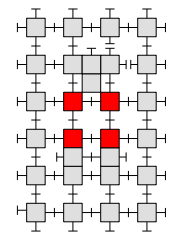

(j)

Figure 9: Details of the Send step of the primitive operation TrAnsfer. 


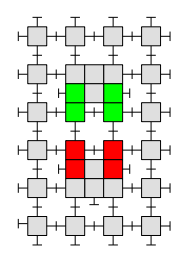

(a)

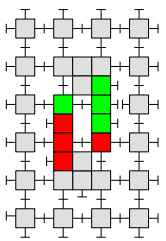

(f)

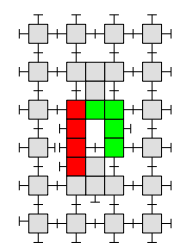

(k)

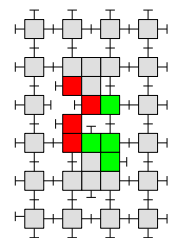

(p)

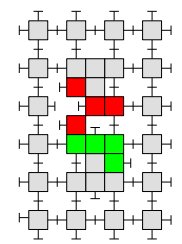

(u)

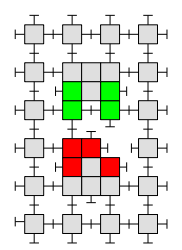

(b)

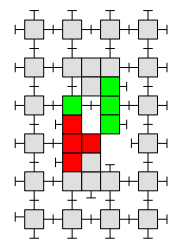

(g)

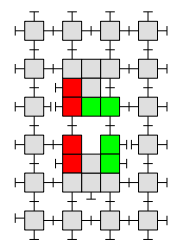

(l)

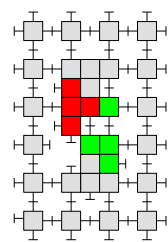

(q)

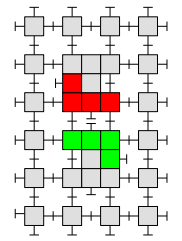

(v)

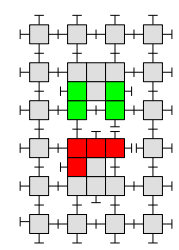

(c)

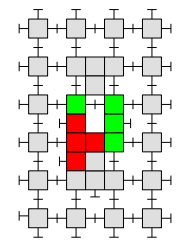

(h)

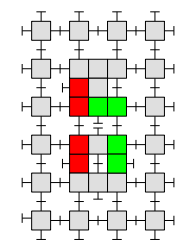

$(\mathrm{m})$

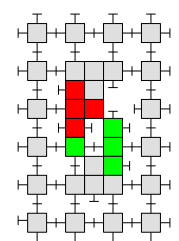

(r)

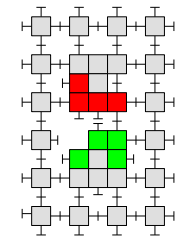

(w)

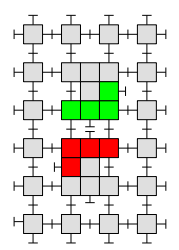

(d)

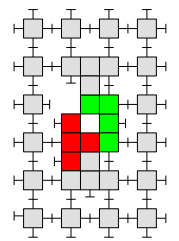

(i)

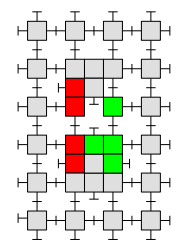

(n)

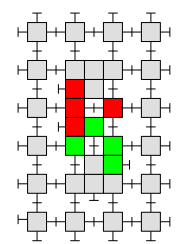

(s)

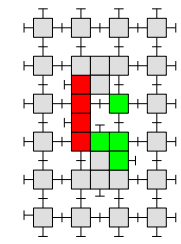

(o)

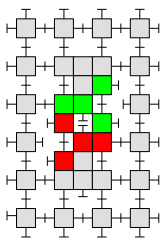

(e)

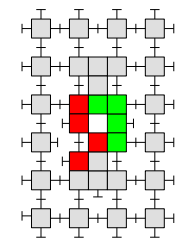

(j)

Figure 10: Details of the Exchange step of the primitive operation TRANSFER. 


\section{SwITCH}

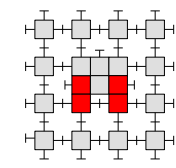

(a)

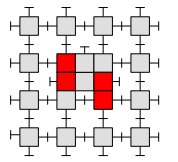

(f)

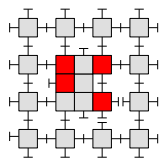

(k)

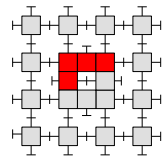

(p)

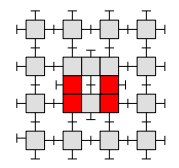

(b)

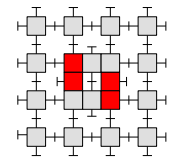

(g)

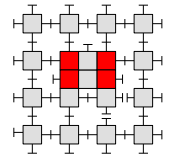

(l)

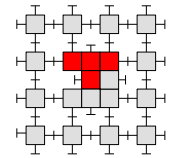

(q)

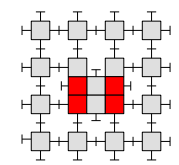

(c)

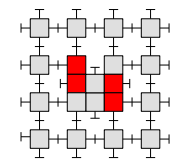

(h)

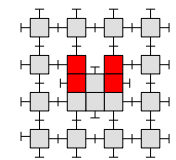

(m)

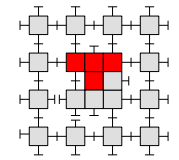

(r)

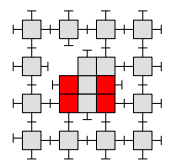

(d)

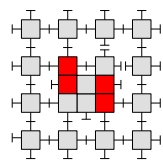

(i)

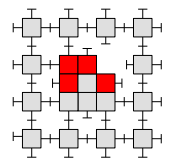

(n)

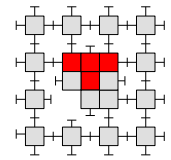

(s)

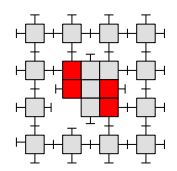

(e)

Figure 11: Details of SwITCH. 